\title{
JOURNAL OF APPLIED PHARMACEUTICAL RESEARCH | JOAPR
}

\section{www.japtronline.com}

ISSN: $2348-0335$

\section{RECENT ADVANCEMENT IN THE METHOD OF TRANSDERMAL DRUG DELIVERY SYSTEM: A REVIEW Sudip Halder*, Prodipta Chakraborty, Darshan Pradhan, Arnab Bagchi}

\author{
Article Information \\ Received: $4^{\text {th }}$ September 2020 \\ Revised: 28 ${ }^{\text {th }}$ March 2021 \\ Accepted: $21^{\text {st }}$ April 2021
}

\section{Keywords}

Transdermal patch,

bioavailability, dermatological, method

\begin{abstract}
A transdermal patch is a medicated adhesive patch that is applied to the skin and used to deliver a particular amount of medication into the bloodstream through the skin. It aids in the recovery of an injured bodily part. The transdermal drug delivery system (TDDS) provides an alternative safe means of drug delivery to previous intrusive techniques. In the past, topically applied lotions and ointments were the most commonly utilised systems for dermatological issues. The fact that some of these formulations cause systemic side effects indicates that they are absorbed through the skin. All topically applied medication formulations intended to transport the active ingredient into the general circulation are included in the transdermal delivery system.

To overcome the skin's formidable barrier to topical medication administration, several substances have been utilised. Because of their unique qualities, such as improved bioavailability, regulated pharmaceutical release, and improved patient compliance, TDDS have recently gained increased attention. The current review focuses on the various advancements in transdermal drug delivery, as well as the various available methods for preparing transdermal patches, characterization and assessment tools for transdermal patch preparation, patents, transdermal compound clinical trials, and drug approved and future applications of transdermal drug delivery systems. As a result, in recent years, the Transdermal Drug Delivery System has received a lot of attention.
\end{abstract}

\section{INTRODUCTION}

The self-contained discrete dosage forms that, when applied to undamaged skin, transport the drug(s) via the skin at a controlled rate to the systemic circulation are known as transdermal therapeutic systems [1]. The most common means of administering most medications is through conventional dosage forms, which are primarily taken orally. However, the disadvantages of traditional dosage forms are that they frequently create substantial range changes in plasma drug concentrations, resulting in either unwanted toxicity or poor

* Department of Pharmaceutics, Himalayan Pharmacy Institute, Majhitar, East Sikkim India 737136

\section{*For Correspondence: arnab.pharmacist@gmail.com}

\section{(C)2021 The authors}

This is an Open Access article distributed under the terms of the Creative Commons Attribution (CC BY NC), which permits unrestricted use, distribution, and reproduction in any medium, as long as the original authors and source are cited. No permission is required from the authors or the publishers. (https://creativecommons.org/licenses/by-nc/4.0/) 
efficacy. Not only that, but there are also additional issues to consider, such as repeated dosing and inconsistent absorption. As a result, the concept of a controlled medication delivery system or therapeutic system was born [2].

The human body's skin is one of the most vast and easily accessible organs. It has a surface area of roughly $2 \mathrm{~m}^{2}$ (or $3000 \mathrm{inch}^{2}$ ) and gets around one-third of the blood that circulates through an ordinary adult's body. Only a few milimeters in thickness (2.970.28 mm). The skin keeps the underlying blood circulation system and vital organs safe from the elements. It acts as a barrier against physical and chemical attacks, as well as protecting the body from microorganism invasion [3].

\section{ADVANTAGES \& DISADVANTAGES OF TRANSDERMAL DRUG DELIVERY [4,5,6,7]}

Compared to other traditional techniques, the transdermal system has some significant advantages

$>$ Because the duration of effect is greater, the frequency of dose is reduced.

$>$ Drugs that require regular doses are more convenient to deliver.

$>$ Aids in the improvement of bioavailability.

$>$ Controls the plasma level uniformity.

$>$ The drug administration can be stopped by simply removing the patch from the skin.

$>$ With a non-invasive, painless, and uncomplicated application, you may be confident in patient compliance and comfort.

Some of the greatest disadvantages of transdermal drug delivery are:

1. There is a small chance of local irritation at the application site.

2. The medicine, the adhesive, or any excipients in the patch formulation can produce erythema, irritation, and local edoema.

\section{METHODS OF PREPARATION OF TRANSDERMAL PATCH [8]}

1. Asymmetric TPX membrane method.

2. Circular Teflon mould method.

3. Mercury substrate method.

4. By using "IPM membranes” method.

5. By using "EVAC membranes” method.

6. Preparation of TDDS by using proliposomes.

7. By using free film method.

\section{Asymmetric TPX membrane method}

The backing membrane for a prototype patch will be a heat sealable polyester sheet (type 1009, 3m) with a $1 \mathrm{~cm}$ diameter concave. The drug sample is dispensed into the concave membrane, which is subsequently sealed with an adhesive and covered by an asymmetric TPX poly (4-methyl-1-pentene) membrane. The dry/wet inversion procedure is used to make them. To make a polymer solution, TPX is dissolved in a mixture of solvent (cyclohexane) and non solvent ingredients at $60^{\circ} \mathrm{C}$. The polymer solution is maintained at $40^{\circ} \mathrm{C}$ for 24 hours before being cast on a glass plate with a Gardner knife to a pre-set thickness. After that, the casting film is evaporated at $50^{\circ} \mathrm{C}$ for 30 seconds, and the glass plate is immediately immersed in the coagulation bath [at $25^{\circ} \mathrm{C}$ ]. The membrane can be removed after 10 minutes of immersion and dried in a circulation oven at $50^{\circ} \mathrm{C}$ for 12 hours [9].

\section{Circular Teflon method}

In an organic solvent, solutions containing polymers in various ratios are utilised. The amount of medicine calculated is dissolved in half the amount of the same organic solvent. Enhancers are dissolved in the other half of the organic solvent and then added in various concentrations. As a plasticizer, diNbutyl phthalate is added to the drug polymer solution. The entire mixture must be stirred for 12 hours before being placed into a circular Teflon mould. In a laminar flow hood model with an air speed of $0.5 \mathrm{~m} / \mathrm{s}$, the moulds should be positioned on a levelled surface and covered with an inverted funnel to manage solvent vaporisation. After 24 hours, the solvent is allowed to evaporate. To eliminate ageing effects, the dried films must be held for another 24 hours at $250.5^{\circ} \mathrm{C}$ in a desiccator containing silica gel before being evaluated. Within one week of their preparation, the type films must be reviewed. [10].

\section{Mercury substrate method}

The medication, along with the plasticizer, is dissolved in a polymer solution. It is then stirred for 10 to 15 minutes to achieve a homogeneous dispersion before being poured into a levelled mercury surface and covered with an inverted funnel to prevent solvent evaporation (Wiechers 1992). Rathore et al. looked at using ethyl cellulose and cellulose acetate polymer to make terbutaline sulphate patches with a transdermal matrix. The terbutaline sulphate transdermal patches were made using a mercury substrate and a solvent casting process. Various polymeric terbutaline sulphate transdermal patches were 
produced in the current study. A permeability enhancer's effect on drug permeability from cellulose acetate and ethyl cellulose patches was studied. The polymeric combinations were capable of creating good films, and the casting technique on mercury substrate resulted in good films. Using Eudragit RL 100, Eudragit RS 100, Polyvinyl pyrollidone (PVP) as polymers, glycerol and propylene glycol as plasticizers, and Span 80 as a permeation enhancer, Patel et al. developed transdermal patches containing glibenclamide (1.06 percent w/v, i.e. $13.5 \mathrm{mg} / \mathrm{cm} 2$ ). At the end of 24 hours, the formulation including Eudragit RL 100 and propylene glycol as a plasticizer had 98.02 percent complete and sustained release [11].

\section{By using “IPM membranes” method}

The drug is mixed with water and propylene glycol containing carbomer 940 polymers and whirled for 12 hours in a magnetic stirrer. With the addition of triethanolamine, the dispersion will be neutralised and viscous. If the drug's solubility in aqueous solution is poor, buffer $\mathrm{pH} 7.4$ can be employed to make a solution gel. The gel that has been created will be integrated into the IPM membrane [12].

\section{Preparation of TDDS by using proliposomes}

A film deposition method and a carrier method are used to create the proliposomes. Lecithin with the ratio of 0.1:2.0 can be used as an improved version of the prior reference drug. $5 \mathrm{mg}$ of mannitol powder is placed in a $100 \mathrm{ml}$ round bottom flask that is kept at $60-70^{\circ} \mathrm{C}$ and spun at $80-90$ rpm for 30 minutes while vacuum drying the mannitol. After drying, the water bath's temperature is set at $20-30^{\circ} \mathrm{C}$. At $37^{\circ} \mathrm{C}$, a $0.5 \mathrm{ml}$ aliquot of the organic solution is placed in the round bottomed flask, and after complete drying, additional $0.5 \mathrm{ml}$ aliquots of the solution are added. The drug-loaded mannitol powders (proliposomes) are placed in a desiccator overnight and sieved through a 100 grit sieve after the final loading. The powder is collected and placed in a glass bottle to be stored at a low temperature until characterisation $[13,14]$.

\section{By using free film method}

Casting on a mercury surface produces a free cellulose acetate film. Chloroform is used to make a 2 percent w/w polymer solution. Plasticizers are used at a 40 percent weight-to-weight ratio in the polymer. Five $\mathrm{ml}$ of polymer solution was placed in a glass ring that was placed over the mercury surface in a glass petri dish. An inverted funnel was placed over the petridish to control the rate of solvent evaporation. Observing the mercury surface after the solvent has completely evaporated reveals the $\mathrm{lm}$ creation. The dried $\mathrm{lm}$ will be separated and stored in desiccators between wax paper sheets until needed. By varying the volume of the polymer solution, free films of various thicknesses can be created [15].

\section{CONCLUSION}

A lot of progress has been achieved in the field of transdermal patches. The transdermal medicine delivery system has piqued the curiosity of many academics due to its multiple benefits. To improve transdermal medication distribution, a variety of techniques have been used, including chemicals, electric fields, and ultrasound These advancements have made transdermal medication delivery a viable option for systemic drug administration. In the last two decades, there has been a tremendous surge in scientific interest in this field. Several studies have been conducted to safely breach the skin's barrier function, allowing therapeutic amounts of medication to be administered. Studies using solution or suspension formulations, on the other hand, have a restricted application potential. Transdermal devices should be designed with the system's functional and relevant characteristics in mind. Through a greater understanding of the physicochemical qualities of the medication, the physiology of skin, the mechanism of action of enhancers, and the interaction between formulation components, future study should be able to ensure improved delivery. Furthermore, a wider range of compounds could be addressed in the transdermal delivery arena with improved device design.

\section{FINANCIAL ASSISTANCE Nil}

\section{CONFLICT OF INTEREST}

The authors declare no conflict of interest.

\section{AUTHOR CONTRIBUTION}

Sudipa Halder, Prodipta Chakraborty \& Darshan Pradhan designed the review. They made necessary corrections \& revised the manuscript. Sudip Halder \& Arnab Bagchi collected the content and reviewed literatures. They also contributed in drafting the manuscript. All the authors framed final manuscript.

\section{REFERENCES}

[1] Kehar S, Stbir S, Arpana R. Formulation and evaluation of matrix type transdermal patch of bisoprolol fumarate by 
mercury substrate method. European Journal of Biomedical and Pharmaceutical Sciences, 5(1), 346-350 (2017)

[2] Prausnitz MR, Langer R. Transdermal drug delivery. Nat Biotechnol 26(11), 1261-8. (2008).

[3] Chien Y.W. Novel drug delivery system. Second edition. New york london. Taylor \& francis group, 6(4), 301-380 (1991)

[4] Bhowmik D, Chiranjib , Margret C, Jayakar B, Sampath K. P. Recent advances in transdermal drug delivery system. International Journal of PharmTech Research, 2(1), 68-77 (2010)

[5] Robinson J. R, Lee H. L. Controlled drug delivery fundamentals and applications. $2^{\text {nd }}$ edition. New York, 524552 (1987)

[6] Aquil M, Sultana Y, Ali A. Matrix type transdermal drug delivery systems of metoprolol tartrate: in vitro characterisation. Acta Pharma, 53, 119-125 (2003)

[7] Singh J, Tripathi K. P, Sakia T. R. Effect of penetration enhancers on the in vitro transport of ephedrine trough rat skin and human epidermis from matrix based transdermal formulations. Drug dev. Ind. Pharm, 19, 1623-1628 (1993)

[8] Sharma N. A Brief Review on Transdermal Patches. Organic and Medicinal Chemistry, 7(2),1-5 (2018)

[9] Ajit K. V, Prabhudutta P, Navneet K. V, Dhaneshwar K. V, Jai N. M. An overview on transdermal patches. International Journal of Pharmacy Review \& Research, 7(1),17-23 (2017)
[10] Ansari K, Singhai A. K, Saraogi G. K. Recent advancement in transdermal drug delivery system. International Journal of Pharmacy and Pharmaceutical Sciences, 3(5), 52-59 (2011)

[11] Alam M. I, Alam N, Singh V, Alam M.S, Ali M. S, Anwer T, Safh M. M. Type, preparation and evaluation of transdermal patch: a review. World journal of pharmacy and pharmaceutical sciences, 2(4), 2199-2233 (2013)

[12] Chauhan A, Kumar K. A comprehensive review on matrix type transdermal patches. World Journal of Advance Healthcare Research, 4(2), 240-245 (2020)

[13] Kumar J. A, Pullakandam N, Prabu S. L, Gopal V. Transdermal drug delivery system : an overview. International Journal of Pharmaceutical Sciences Review and Research, 3(2), 49-54 (2010)

[14]Bhairam M, Roy A, Bahadur S, Banafar A, Patel M, Turkane D. Transdermal drug delivery system with formulation and evaluation aspects: Overview. Res. J. Pharm. Technol., 5, 1168-76 (2012).

[15] Kharia A, Gilhotra R, Singhai A. K. Overview of Transdermal Medicated Patches with its research updates in preceding years. Journal of Drug Delivery and Therapeutic, 9(3),1094-1102 (2019) 